0\&rd=1\&h=DvMnugNmFXffO88ET_7MVMcn6uXYrh_Ib7-Ju-Si-

es\&v=1\&r=https $\% 3 \mathrm{a} \% 2 \mathrm{f} \% 2$ fgithub.com $\% 2 \mathrm{fzzet} \% 2 \mathrm{fRestApiTutorial.ru} \% 2 \mathrm{fblob} \% 2 \mathrm{fmaster} \% 2 \mathrm{fhttpstatuscodes} . \mathrm{html} \& \mathrm{p}=\mathrm{D}$ evEx,5040.1.

[5] Lastnitescurry, "lastnitescurry/j2ee-rest-jersey-jetty-tomcat-maven-jenkinsfile [Електронний pecypc] // GitHub. - 2016. Режим доступу до ресурсу: https://github.com/lastnitescurry/j2ee-rest-jersey-jetty-tomcat-maven-jenkinsfile.

УДК 681.513 .7

\title{
НАСТРОЙКА НЕЙРОННОЙ СЕТИ ПРИ АВТОМАТИЧЕСКОМ СИНТЕЗЕ СЕТЕЙ ПЕТРИ
}

\author{
Гурский А.А. ${ }^{1}$, Дубна С.М.
}

Институт компьютерных систем и технологий им. П.Н. Платонова Одесской национальной академии пищевых технологий, г. Одесса

${ }^{1}$ ORCID: http://orcid.org/0000-0001-5158-2125

1'E-mail: Gurskiya2017@gmail.com

Copyright $\mathbb{C} 2017$ by author and the journal "Automation technological and business - processes". This work is licensed under the Creative Commons Attribution International License (CC BY). http://creativecommons.org/licenses/by/4.0/

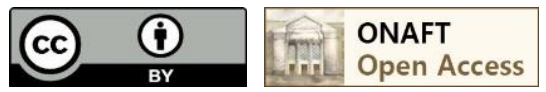

Аннотация: В настоящей работе представлен определенный этап разработки интеллектуальной системы, связанной с автоматическим синтезом сетей Петри. Рассматривается определенная архитектура искусственной нейронной сети, которая положена в основу интеллектуальной системbl, направленной главным образом на формирование алгоритмов настройки координирующих систем автоматического управления. Особенность функционирования рассматриваемой архитектуры нейронной сети заключается в том, что в любой момент времени может быть активен только один нейрон из определенного количества возможных для активизации других нейронов сети. В работе представляется алгоритм настройки данной нейронной сети, который связан с инцидентной матрицей формируемой сети Петри. При этом формируемая сеть Петри представляет алгоритм настройки координирующей системы автоматического управления.

В заключительной части работы представлена разработанная в программной среде MATLAB/Simulink cuстема, формирующая инцидентную матрицу сети Петри на базе функционирования нейронной сети. Отражается визуализаџия прочесса формирования сети Петри, представляющей алгоритм настройки системы управления. Данная визуализация дает возможность представить результат формирования алгоритма, тем самым позволяет определить специалисту, при необходимости, нужную корректировку данного алгоритма.

Abstract: The certain stage in the development of the artificial intelligent system is considered in this paper. This intelligent system associates with the automatic synthesis and the composition of Petri nets. The intelligent system consisting of the certain architecture of the neural network is considered. This intelligent system is designed to develop and generate algorithms for tuning the coordinating systems of automatic control. The peculiarity of the neural network architecture lets to activate one neuron of possible at any moment of time. In this case the activity of one neuron causes the activation of other neurons in the network. The algorithm for tuning this neural network is presented in this scientific work. This tuning algorithm associates with the incident matrix formed by Petri net. The Petri net represents the algorithm for tuning of the coordinating systems of automatic control.

The system designed in the MATLAB / Simulink software environment is presented in the final part of the scientific work. This system forms the incident matrix of the Petri net during the functioning of the neural network. The visualization of the process of the Petri net formation is presented. This visualization of the Petri net provides the opportunity to find out the result of the algorithm formation. The specialist can determine the necessary adjustment of the algorithm for tuning of the coordinating systems of automatic control. The attempt was made to use principle of reinforcement learning in this artificial intelligence system. This principle of reinforcement learning represents the actively developing field of the artificial intelligence, neural network modeling and control. 
Ключевые слова: Нейронная сеть, сеть Петри, координирующая система, обучение с подкреплением, интеллектуальная система, автоматический синтез сети Петри

Keywords: Neural network, Petri net, coordinating system, training with reinforcement, intelligent system, automatic synthesis of Petri net

\section{Введение}

Искусственная нейронная сеть как вычислительная схема, включающая множество вычислительных единиц нейронов, может представить некоторую интеллектуальную систему при наличии определенного метода изменения коэффициентов межнейронных связей. Именно подобное изменение коэффициентов межнейронных связей, которое имеет место в процессе обучения нейронной сети, может сыграть важную роль в проявлении интеллектуальных особенностей нейронной сети при решении определенных задач. В частном случае такие задачи могут быть связаны с автоматическим синтезом сетей Петри, а также с синтезом алгоритмов управления некоторых объектов [1,2].

Актуальность темы

Автоматический синтез и композиция сетей Петри предполагает использование соответствующих определенных методов. Однако, для того чтобы формируемая сеть Петри была применима для решения определенной задачи, необходимо применение интеллектуальных технологий, способных заменить эксперта в области формирования определенных алгоритмов. Например, в настоящем случае - замена эксперта в области формирования алгоритмов настройки различных координирующих систем автоматического управления.

Теория синтеза координирующих систем автоматического управления имеет существенную историю развития, большой вклад в которую внесли М.Б. Игнатьев (1963), В.А. Романов (1964), Л.М. Бойчук (1975 - 1995$)$ и др. [3]. Однако координирующие системы автоматического управления не имеют широкого распространения в технике в связи с нетрадиционной целью управления. В связи с этим развитие некоторых направлений синтеза координирующих систем актуально, особенно в области траекторных задач координирующего управления [4], и также актуально развитие интеллектуальной системы, позволяющей заменить эксперта в области настройки соответствующих систем управления.

Соответственно, разработка интеллектуальной системы, связанной с автоматическим синтезом сетей Петри, представляющих алгоритмы настройки координирующих систем управления, является актуальной. В данном случае автоматически синтезируемая сеть Петри дает возможность представить специалисту соответствующей области результат формирования алгоритма настройки системы управления, а также, при необходимости, дает возможность определить необходимую коррекцию данного алгоритма..

Анализ существующих разработок и исследований в области автоматического синтеза и композиции сетей Петри

Возможность автоматического синтеза и существование методов автоматического построения сетей Петри отмечались в работе Джеймса Питерсона [5]. С тех пор появился ряд научных публикаций, связанных с автоматической генерацией и композицией сетей Петри, в которых отражаются особенности построения сетей Петри, основанные на определенных методах $[1,6,7]$. Аналогичным образом появилась работа [8], в которой автоматический синтез сетей Петри осуществляется на основе метода проверки достижимости дискретно-непрерывных сетей [9]. Если метод проверки достижимости некоторого состояния в пространстве переменных связан с преобразованием дискретно-непрерывной сети до одного перехода (элемента сети Петри), то при автоматическом синтезе сети Петри процесс обратный, из одного перехода преобразуется сеть Петри, которая представляет достижимость некоторого состояния гибридной системы [9]. Однако исключение эксперта в построении сети Петри и определении некоторого алгоритма управления представляет отсутствие какого либо прикладного характера синтезированной сети Петри. В таком случае в работе [10] была предложена автоматическая композиция сети Петри на базе функционирования нейронной сети, которая представляет интеллектуальную технологию в определении алгоритма настройки координирующей системы автоматического управления. В настоящей работе представляется, неопределенный ранее в работе [10] целесообразный алгоритм настройки нейронной сети, применяемый при автоматическом синтезе сети Петри.

Цель и задача настоящей работы.

Целью настоящей работы является снижение времени на определение алгоритма настройки системы управления при помощи применения интеллектуальной системы, основанной на базе искусственной нейронной сети, осуществляющей автоматический синтез сетей Петри.

Для достижения поставленной цели в рамках настоящей работы необходимо определить алгоритм настройки нейронной сети, непосредственно связанный с математическим описанием синтезируемой сети Петри, представляющей алгоритм настройки системы управления.

Особенности функционирования нейронной сеть при автоматической генерации сети Петри

Первоначально было установлено, что автоматическая генерация сети Петри должна выполняется при функционировании нейронной сети определяющей интеллектуальную технологию формирования алгоритма управления некоторым объектом.

Данная нейтронная сеть является многослойной и прямонаправленной. Каждый слой нейронной сети несет определенную функциональную нагрузку. Первый слой нейронной сети, обеспечивает классификацию возможных 
http://www.atbp.onaft.edu.ua/

алгоритмов. Внутренний слой определяет алгоритмы управления. Выходной слой обеспечивает формирование определенной матрицы инцидентности сети Петри, представляющей алгоритм управления объектом. Как показано на рисунке 1, нейронную сеть можно разделить на секторы, каждый сектор которой представляет определенный алгоритм управления и соответствующую матрицу инцидентности сети Петри. Так как формирование алгоритма представляется как пошаговый - поэтапный процесс, то каждый шаг при формировании алгоритма сопровождается активизацией определенного нейрона, называемого исходным. Исходные нейроны W содержатся в каждом секторе. Перенастройка весовых коэффициентов межнейронных соединений, связанных с исходным нейроном, влечет за собой корректировку соответствующего алгоритма, если он не удовлетворяет определенным требованиям

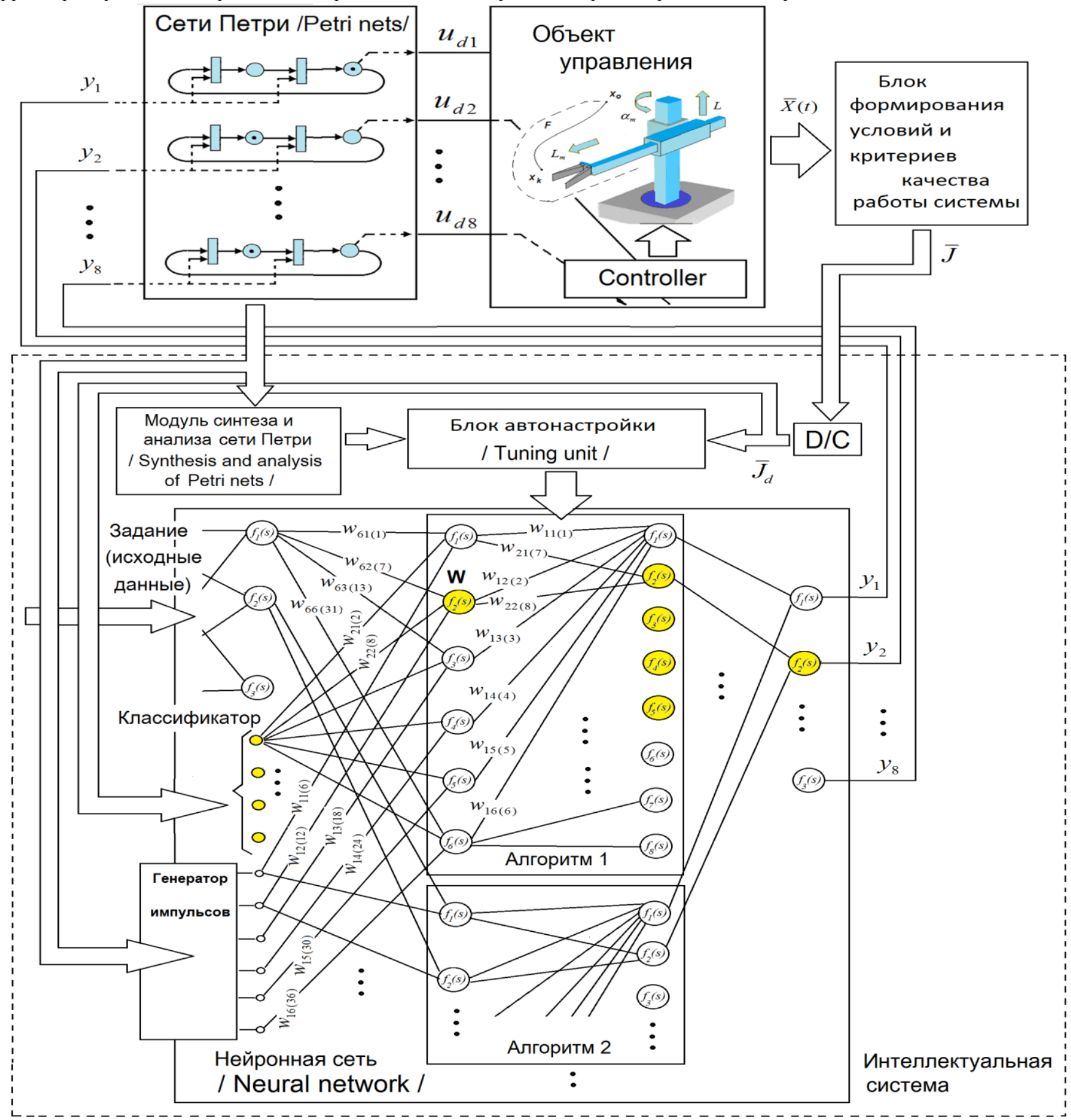

Рис. 1 - Упрощенная структурная схема системы, формирующей алгоритм управления при автоматической композиции сети Петри на базе функционирования искусственной нейронной сети

Нейронная сеть как вычислительная схема определяет матрицу инцидентности сети Петри набором коэффициентов межнейронных соединений , связанных с исходными нейронами. Матрица инцидентности при рассматриваемой схеме, представленной на рисунке 1 , имеет следующий вид: 


\begin{tabular}{|c|c|c|c|c|c|c|c|}
\hline \multirow{2}{*}{\multicolumn{2}{|c|}{85}} & \multicolumn{5}{|c|}{$\begin{array}{c}\text { Автоматизація технологічних і бізнес-процесів Volume 10, Issue } 1 \text { /2018 } \\
\text { http://www.atbp.onaft.edu.ua/ }\end{array}$} & \multirow{2}{*}{29} \\
\hline & & $t_{1}$ & $t_{2}$ & $t_{3}$ & $t_{4}$ & $t_{5}$ & \\
\hline \multirow{8}{*}{$|\boldsymbol{W}|$} & $p_{0}$ & $w_{11(1)}$ & $w_{12(2)}$ & $w_{13(3)}$ & $w_{14(4)}$ & $w_{15(5)}$ & $w_{16(\sigma)}$ \\
\hline & $p_{1}$ & $w_{21(7)}$ & $w_{22(8)}$ & $w_{23(9)}$ & $w_{24(10)}$ & $w_{25(11)}$ & $w_{26(12)}$ \\
\hline & $p_{2}$ & $w_{31(13)}$ & $w_{32(14)}$ & $w_{33(15)}$ & $w_{34(16)}$ & $w_{35(17)}$ & $w_{36(18)}$ \\
\hline & $=p_{3}$ & $w_{41(19)}$ & $w_{42(20)}$ & $w_{43(21)}$ & $w_{44(22)}$ & $w_{45(23)}$ & $w_{46(24)}$ \\
\hline & $p_{4}$ & $w_{51(25)}$ & $w_{52(26)}$ & $w_{53(27)}$ & $w_{54(28)}$ & $w_{55(29)}$ & $w_{56(30)}$ \\
\hline & $P_{5}$ & $w_{61(31)}$ & $w_{62(32)}$ & $w_{63(33)}$ & $w_{64(34)}$ & $w_{65(35)}$ & $w_{66(36)}$ \\
\hline & $p_{6}$ & $w_{71(37)}$ & $w_{72(38)}$ & $w_{73(39)}$ & $w_{74(40)}$ & $w_{75(41)}$ & $w_{76(42)}$ \\
\hline & $p_{7}$ & $w_{81(43)}$ & $w_{82(44)}$ & $w_{83(45)}$ & $w_{84(46)}$ & $w_{85(47)}$ & $w_{86(48)}$ \\
\hline
\end{tabular}

Количество столбцов матрицы инцидентности определяется количеством исходных нейронов, а количество строк определяется количеством выходных нейронов n-го (выходного) слоя нейронной сети.

Определенные условия срабатывания соответствующих переходов сети Петри определяются весовыми коэффициентами входных соединений исходных нейронов. Набор весовых коэффициентов входных соединений исходных нейронов также можно представить в виде следующей матрицы:

$$
\begin{array}{lllllll} 
& t_{1} & t_{2} & t_{3} & t_{4} & t_{5} & t_{6} \\
N_{1} & w_{11(6)} & w_{12(12)} & w_{13(18)} & w_{14(24)} & w_{15(30)} & w_{16(36)} \\
N_{2} & w_{21(2)} & w_{22(8)} & w_{23(14)} & w_{24(20)} & w_{25(26)} & w_{26(32)} \\
N_{3} & w_{31(3)} & w_{32(9)} & w_{33(15)} & w_{34(21)} & w_{35(27)} & w_{36(33)} \\
N_{4} & w_{41(4)} & w_{42(10)} & w_{43(16)} & w_{44(22)} & w_{45(28)} & w_{46(34)} \\
N_{6} & w_{61(1)} & w_{52(11)} & w_{53(17)} & w_{54(23)} & w_{55(29)} & w_{56(35)} \\
& & w_{63(13)} & w_{64(19)} & w_{65(25)} & w_{66(31)}
\end{array}
$$

В данной матрице количество строк N1 ... N6 соответствует количеству различным условиям срабатывания переходов, а количество столбцов соответствует количеству шагов формирования алгоритма.

Согласно рисунку 1, процесс формирования элементов матрицы инцидентности сети Петри осуществляется на базе выходных сигналов у1...у8 нейронной сети, обеспечивающих параллельное - синхронное функционирование соответствующих сетей Петри. Это дает возможность представить некоторую композицию из соответствующих сетей Петри, отражающую алгоритм управления определенным объектом [10]. Если алгоритм управления объектом не соответствует заданным требованиям, при которых осуществляется соответствующее изменение значений критериев качества работы системы, то блок автонастройки /Tuning unit/ должен изменить определенные коэффициенты межнейронных соединений согласно инцидентной матрицы синтезированной сети Петри.

Особенности архитектуры нейронной сети, осуществляющей синтез сетей Петри и формирование алгоритмов управления объектов.

Исходя из вышерассмотренной схемы, представленной на рисунке 1, можно выделить некоторую определенную архитектуру нейронной сети, которая может применяться при формировании алгоритма управления, отраженного соответствующей сетью Петри. Данная архитектура нейронной сети, изображенная на рисунке 2 , представляется двухслойной с обратными связями и с элементами задержки сигналов.

Особенность функционирования такой нейронной сети заключается в том, что в любой момент времени может быть активен только один нейрон из $\mathrm{n}$ - возможных нейронов во входном слое. При этом активизация лишь одного нейрона во входном слое может вызвать активизацию $\mathrm{N}$ - нейронов в выходном слое.

Расчет синаптических коэффициентов исходных нейронов вычисляется на основе синаптических коэффициентов входных соединений выходных нейронов сети по следующей формуле:

$$
w_{n k(u)}=w_{n k} \cdot\left(\left|b_{k(u)}\right|-\sum_{n=1}^{m} w_{n k}\right)+w_{n k}-1
$$

где: синаптический коэффициент n-го входа входного k-го нейрона; синаптический коэффициент k-го входа выходного n-го нейрона; величина смещения входного k-го нейрона. 


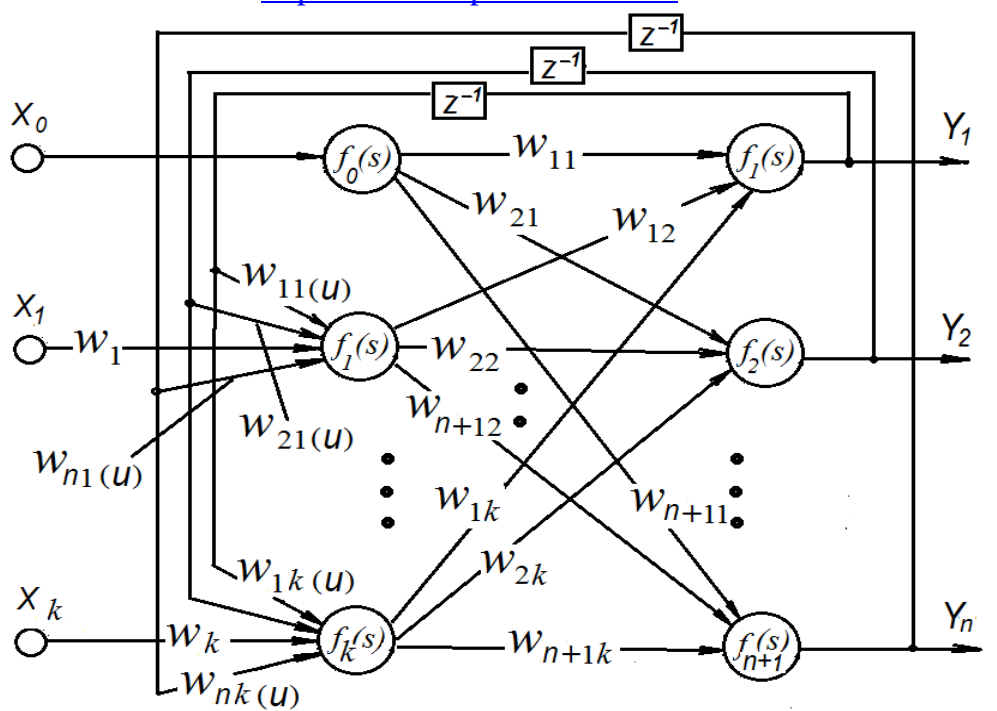

Рис. 2 - Архитектура нейронной сети, используемая при формировании инцидентной матрицы сети Петри

Данная формула расчета коэффициентов межнейронных связей определена из условия активизации лишь одного входного нейрона из $\mathrm{n}$ - возможных нейронов, в любой момент времени. Количество выходных нейронов сети должно быть такое, чтобы не повторялись комбинации значений сигналов выходов Y1 ... Yn.

Для проверки принципиальной пригодности рассмотренной архитектуры нейронной сети в программной среде MATLAB \Simulink была реализована соответствующая схема, представленная на рисунке 3 . Как видно из рисунка 3 представлена схема нейронной сети, состоящей из 3-х входных и 3-х выходных нейронов и имеющей обратные связи с элементами задержки сигнала.

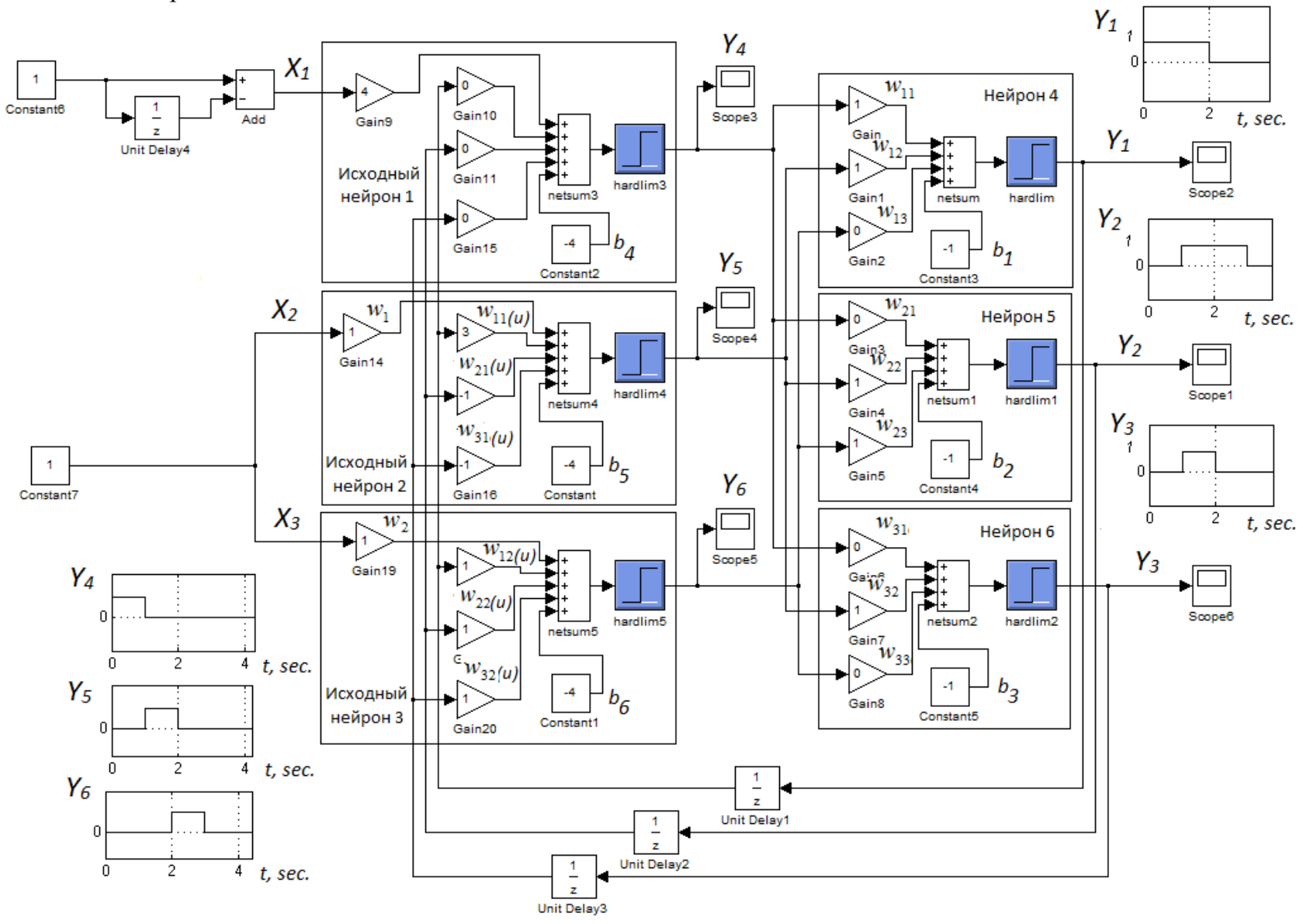

Рис. 3 - Структурная схема нейронной сети с исходными нейронами

Согласно вышеприведенной формуле представим следующие расчеты некоторых коэффициентов межнейронных соединений соответствующей нейронной сети: 


$$
\begin{gathered}
\text { http://www.atbp.onaft.edu.ua/ } \\
w_{32(u)}=w_{32} \cdot\left(\left|b_{2(u)}\right|-\sum_{n=1}^{3} w_{n 2}\right)+w_{32}-1=1 \cdot(|-4|-(1+1+1))+1-1=1 \\
w_{31(u)}=w_{31} \cdot\left(\left|b_{1(u)}\right|-\sum_{n=1}^{3} w_{n 1}\right)+w_{31}-1=1 \cdot(|-4|-(1+0+0))+1-1=3
\end{gathered}
$$

Из соответствующих временных диаграмм активизаций нейронов, представленных также на рисунке 3 , видно, что в любой момент времени активен только один нейрон из 3-х входных, при этом количество активных выходных нейронов различно. Так как в каждый момент времени активен лишь один нейрон во входном слое, то расчеты коэффициентов межнейронных соединений были выполнены верно.

Алгоритм настройки нейронной сети

Рассматриваемый алгоритм настройки нейронной сети соответствует алгоритму обучения с последовательным подкреплением знаний, при котором сети не предоставляются желаемые значения выходных сигналов, а вместо этого сети ставится оценка, хорош выходной сигнал или плох [11].

Предполагается, что в нейронной сети в определенный момент времени может быть активен лишь один нейрон, называемый исходным, из n возможных нейронов в слое. Исходя из этого, сущность настройки нейронной сети можно отразить следующим образом: если активность исходного нейрона привела к нежелательной ситуации, то связи с этим нейроном W должны претерпеть изменения (рис. 4). При этом изменение связей с исходным нейроном должно происходить таким образом, чтобы не нарушить правила формирования сети Петри.

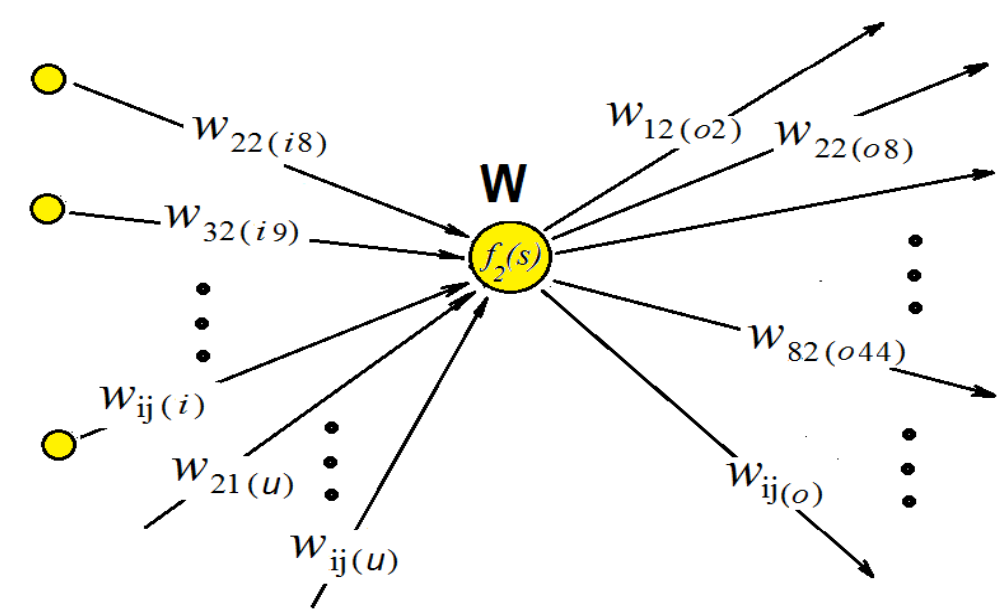

Рис. 4 - Схема, отражающая связи исходного нейрона с обозначенными весами межнейронных соединений.

При учете правил формирования сети Петри коэффициенты межнейронных соединений, связанных с исходным нейроном, должны измениться определенным образом в зависимости от сформированной матрицы инцидентности. Итак, если при активизации определенных нейронов была получена нежелательная реакция некоторой системы, то весовые коэффициенты связей этих нейронов wij следует изменить следующим образом:

$$
\begin{aligned}
& w_{i j(o)}=\eta \cdot\left(\sum_{j=1}^{n} w_{i j} \cdot S_{i m}+\left(1-\sum_{j=1}^{n} w_{i j}\right) \cdot f_{i k}\right) \cdot F_{i} ; \quad w_{i j(o)}=\eta \cdot\left(\sum_{j=1}^{n} w_{i j} \cdot S_{i m}+\left(1-\sum_{j=1}^{n} w_{i j}\right) \cdot f_{i k}\right) \cdot F_{i} \\
& F_{i}=\left\{\begin{array}{lll}
1 & n p u & \left(\Sigma \bar{w}_{i j}^{T} \cdot \bar{S}_{i m}\right) \cdot\left(1-\Sigma \bar{w}_{i j}^{T}\right) \cdot \bar{f}_{i k}>0 \\
0 & n p u & \left(\Sigma \bar{w}_{i j}^{T} \cdot \bar{S}_{i m}\right) \cdot\left(1-\Sigma \bar{w}_{i j}^{T}\right) \cdot \bar{f}_{i k}=0
\end{array} F_{i}=\left\{\begin{array}{lll}
1 & n p u & \left(\Sigma \bar{w}_{i j}^{T} \cdot \bar{S}_{i m}\right) \cdot\left(1-\Sigma \bar{w}_{i j}^{T}\right) \cdot \bar{f}_{i k}>0 \\
0 & n p u & \left(\Sigma \bar{w}_{i j}^{T} \cdot \bar{S}_{i m}\right) \cdot\left(1-\Sigma \bar{w}_{i j}^{T}\right) \cdot \bar{f}_{i k}=0
\end{array}\right.\right. \\
& w_{i j(i)}=\eta \cdot n_{i h} ;
\end{aligned}
$$

где:

$$
\begin{array}{cccccc} 
& t_{1} & t_{2} & \ldots & t_{j} & \sum \bar{w} \\
p_{0} & w_{11} & w_{12} & \ldots & w_{1 j} & \sum w_{1 j} \\
p_{1} & \ldots & w_{22} & \ldots & w_{2 j} \Rightarrow & \sum w_{2 j} \\
\ldots & \ldots & \ldots & \ldots & \ldots & \ldots
\end{array}
$$


сформированный вектор $\Sigma \bar{w}=\left|\begin{array}{lll}\Sigma w_{1 j} & \ldots . & \Sigma w_{i j}\end{array}\right|^{T} ; \Sigma w_{i j}-$ сумма всех значений $i$-й строки матрицы $|W|$;

$\bar{S}_{i m}, \bar{f}_{i k}-$ вектора разрешенных комбинаций весов межнейронных соединений, не нарушающих правила формирования сети Петри;

$\begin{array}{rcccccccccccc} & \bar{S}_{i 1} & \bar{S}_{i 2} & \bar{S}_{i 3} & & \bar{f}_{i 1} & \bar{f}_{i 2} & \bar{f}_{i 3} & \bar{f}_{i 4} & \bar{f}_{i 5} & \bar{f}_{i 6} & \bar{f}_{i 7} & \bar{f}_{i 8} \\ s_{1 m} & 1 & 0 & 1 & f_{1 k} & 1 & 0 & 0 & 0 & 0 & 0 & 0 & 0 \\ s_{2 m} & 1 & 1 & 0 & f_{2 k} & 0 & 1 & 0 & 0 & 0 & 0 & 0 & 0 \\ s_{3 m} & 1 & 1 & 0 & f_{3 k} & 0 & 0 & 1 & 0 & 0 & 0 & 0 & 0 \\ S_{1} \mid=s_{4 m} & 1 & 0 & 1, & \left|f_{1}\right|=f_{4 k} & 0 & 0 & 0 & 1 & 0 & 0 & 0 & 0 ; \\ s_{5 m} & 1 & 0 & 1 & f_{5 k} & 0 & 0 & 0 & 0 & 1 & 0 & 0 & 0 \\ s_{6 m} & 1 & 1 & 0 & f_{6 k} & 0 & 0 & 0 & 0 & 0 & 1 & 0 & 0 \\ s_{7 m} & 1 & 1 & 0 & f_{7 k} & 0 & 0 & 0 & 0 & 0 & 0 & 1 & 0 \\ s_{8 m} & 1 & 0 & 1 & f_{8 k} & 0 & 0 & 0 & 0 & 0 & 0 & 0 & 1 \\ & & & & & & & & & & & & \\ & \bar{S}_{i 1} & \overline{S_{i 2}} & \bar{S}_{i 3} & & \bar{f}_{i 9} & \bar{f}_{i 10} & \bar{f}_{i 11} & \bar{f}_{i 12} & \bar{f}_{i 13} & \bar{f}_{i 14} & \bar{f}_{i 15} & \bar{f}_{i 16} \\ s_{1 m} & 1 & 0 & 1 & f_{1 k} & 0 & 0 & 0 & 0 & 0 & 0 & 0 & 0 \\ s_{2 m} & 1 & 1 & 0 & f_{2 k} & 1 & 0 & 0 & 1 & 0 & 0 & 0 & 0 \\ s_{3 m} & 1 & 1 & 0 & f_{3 k} & 0 & 1 & 1 & 0 & 0 & 0 & 0 & 0 \\ s_{4 m} & 1 & 0 & 1 & \left|f_{2}\right|=f_{4 k} & 0 & 1 & 0 & 1 & 0 & 1 & 0 & 1\end{array} ;$

$\bar{n}_{i h}-$ вектор комбинации весовых коэффициентов входных связей исходного нейрона;

$$
\left|N_{1}\right|=\begin{array}{ccccc} 
& \bar{n}_{i 1} & \bar{n}_{i 2} & \bar{n}_{i 3} & \bar{n}_{i 4} \\
n_{1 h} & 1 & 0 & 0 & 0 \\
n_{2 h} & 0 & 1 & 0 & 0 \\
n_{3 h} & 0 & 0 & 1 & 0 \\
n_{4 h} & 0 & 0 & 0 & 1
\end{array}
$$

Представленные выше вектора возможных комбинаций синаптических коэффициентов связей нейронов позволяют нейронной сети в процессе функционирования выполнить композицию лишь определенных сетей Петри, так как не все возможные комбинации коэффициентов межнейронных связей представлены. Очевидно, что данные сети Петри могут отражать сугубо определённые алгоритмы управления объектами или, в частном случае, алгоритмы настройки определенных систем управления. Однако следует отметить, что рассматриваемая архитектура нейронной сети, используемая при автоматическом синтезе сетей Петри, имеет определенную особенность необходимую при формировании различных алгоритмов управления

Анализ результатов автоматического синтеза сети Петри при автоматической настройке координирующей системы управления

Для проверки принципиальной пригодности определенного метода настройки нейронной сети, предназначенной для выполнения задачи автоматического синтеза сети Петри, была разработана логическая схема в среде MATLABISimulink, которая генерирует в конечном варианте некоторую матрицу инцидентности [10]. Данная логическая схема, представленная на рисунке 5 , отражает основные функциональные блоки, которые в структурном единстве представляют систему, осуществляющую автоматическую композицию сети Петри, аналогично системе, упрощенная структурная схема которой представлена на рисунке 1 . Как видно из рисунка 5 , алгоритмы настройки нейронной сети и модель функционирования сетей Петри реализованы на базе Stateflow-диаграмм, а слои нейронной сети, содержащие исходные нейроны, сформированы в блоках subsystem. 
http://www.atbp.onaft.edu.ua/

Процесс функционирования логической схемы связан с настройкой некоторой координирующей системы (КС) [3, 4], закон управления которой имеет следующий вид:

$$
\bar{u}=\bar{u}_{q}+\bar{u}_{p}=\left[\begin{array}{l}
k_{1} \cdot\left(1+k_{11} \cdot p\right) \cdot \psi \\
k_{2} \cdot\left(1+k_{21} \cdot p\right) \cdot \psi
\end{array}\right]+\left[\begin{array}{c}
0 \\
k_{3} \cdot\left(1+k_{31} \cdot p\right) \cdot e_{1}
\end{array}\right]
$$

где: $e_{1}-$ сигнал рассогласования; $\psi$ - отклонение от соотношения переменных; $p=d / d t-$ оператор дифференцирования; $k_{1}, k_{2}, k_{3}$ - параметры настройки, которые поочередно изменятся в зависимости от маркировки $X_{d}\left(t_{k}\right)=\left[\mu\left(p_{0}\right), \mu\left(p_{1}\right), \ldots \mu\left(p_{7}\right)\right]^{T}$ формируемой сети Петри. Наличие маркера в позиции $p_{i}$, где $i=$ $0 \ldots 7$, отражается наличием единичного сигнала на соответствующем выходе Stateflow - диаграммы. При этом, если позиция $p_{i}$ имеет маркер, то происходит процесс увеличения значения параметра настройки $k_{j}$ до экстремального значения показателя $J_{i}$ качества работы КС, то есть $\mu\left(p_{i}\right)=1 \Rightarrow \uparrow k_{j}$, таким образом $\mu\left(p_{1}\right)=1 \Rightarrow \uparrow k_{1} ; \mu\left(p_{2}\right)=1 \Rightarrow \downarrow k_{1} ;$ $\mu\left(p_{3}\right)=1 \Rightarrow \uparrow k_{2} ; \mu\left(p_{4}\right)=1 \Rightarrow \downarrow k_{2} ; \mu\left(p_{5}\right)=1 \Rightarrow \uparrow k_{3} ; \mu\left(p_{6}\right)=1 \Rightarrow \downarrow k_{3}$.

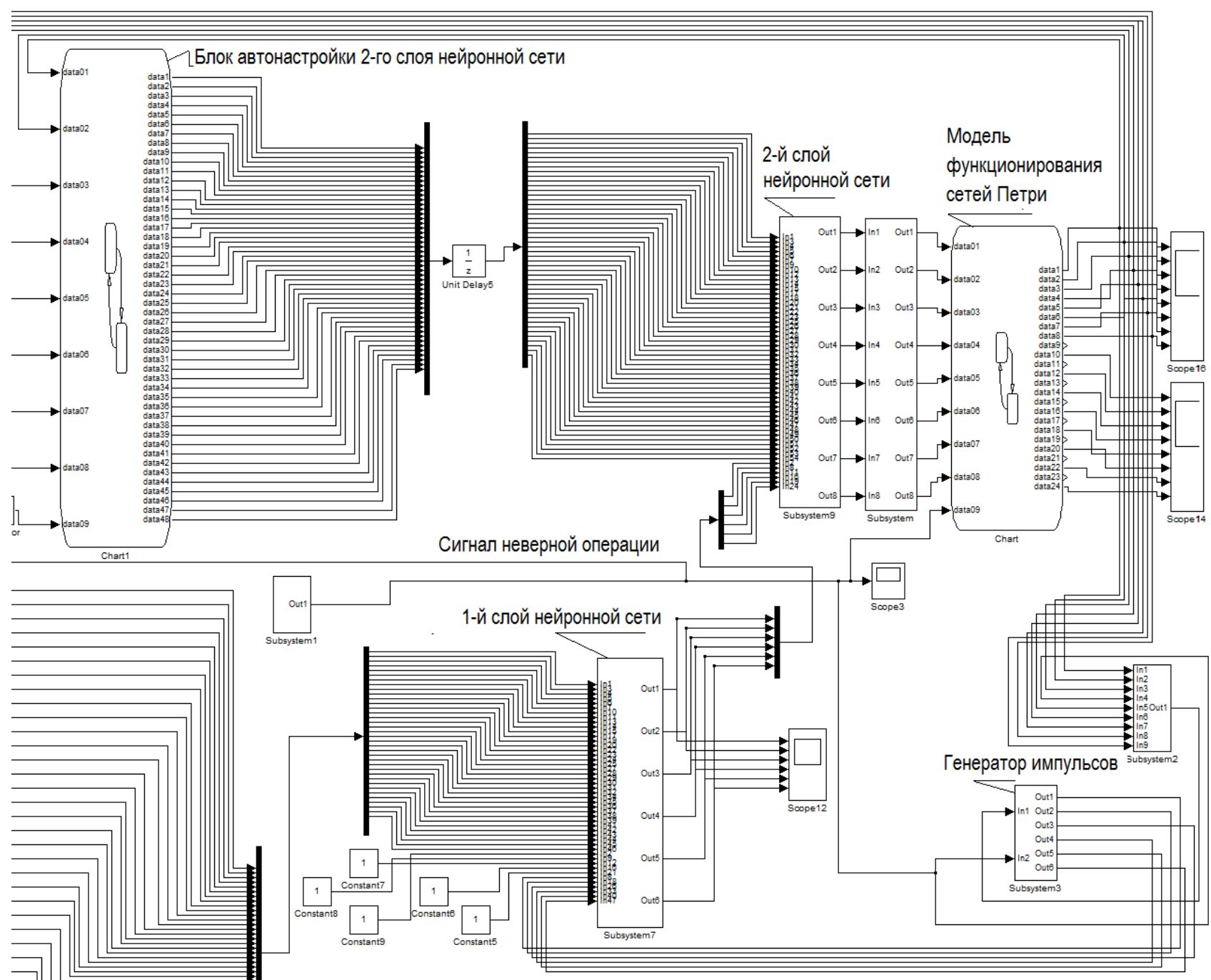

Рис. 5 - Структурная схема системы, формирующей сеть Петри на базе функционирования искусственной нейронной сети

Процесс функционирования нейронной сети системы, структурная схема которой представлена на рисунке 5, сопровождается формированием значений матрицы инцидентности сети Петри. При этом данная матрица инцидентности формируются при движении маркеров сетей Петри, модель функционирования которых представлена в виде Stateflow-диаграмм. На рисунке 6 представлен процесс формирования матрицы инцидентности сети Петри, представляющий алгоритм настройки координирующей системы автоматического управления (КСАУ). Из рисунка 6 видно, что комбинации значений сигналов в определенные моменты времени одинаковые, это свидетельствует о том, что сеть Петри, представляющая алгоритм настройки системы управления, перестраивалась при наличии сигналов 
«неверных операций» и, соответственно, при этом изменялись коэффициенты соответствующих межнейронных связей.

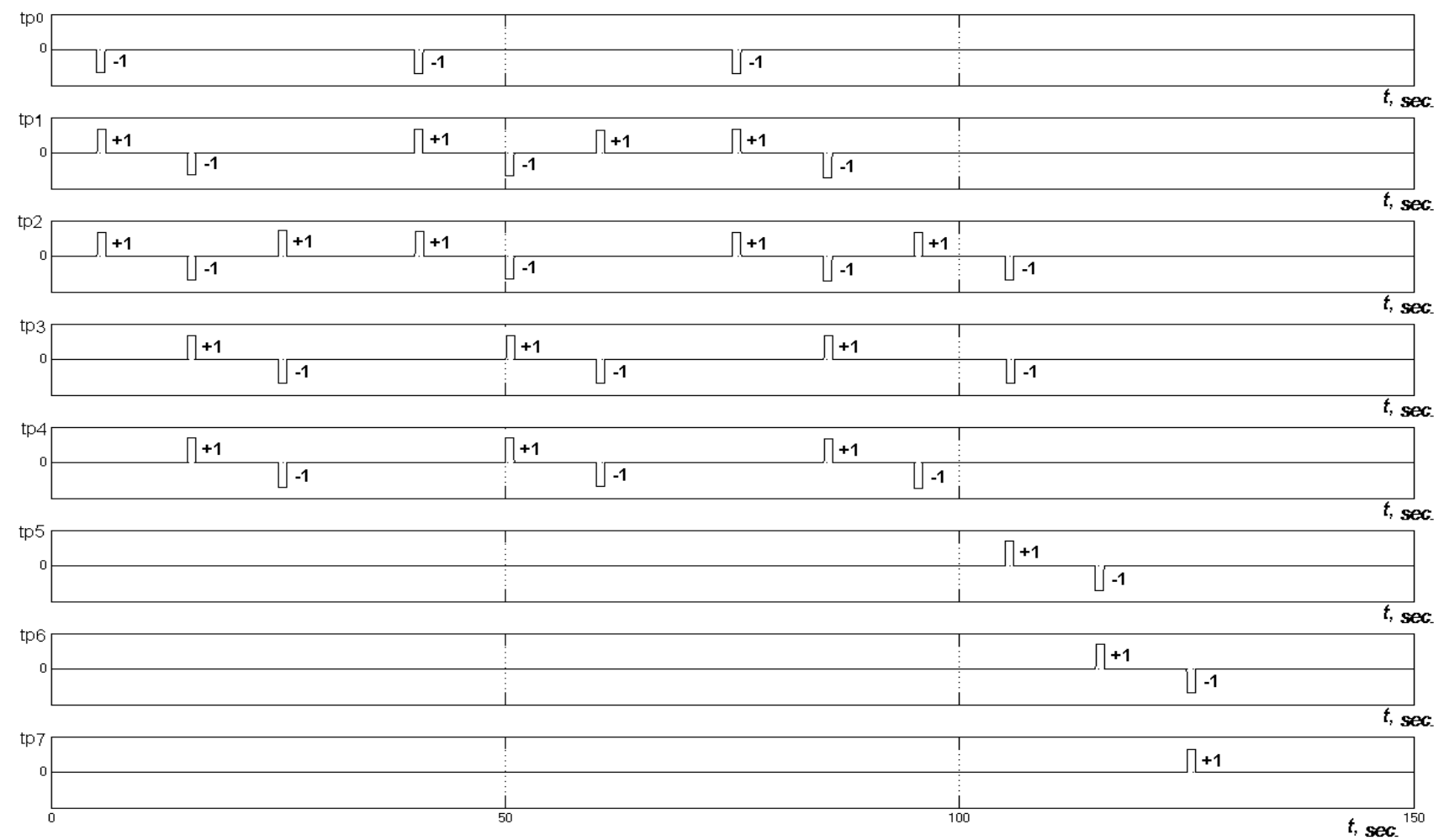

Рис. 6 - Процесс формирования матрицы инцидентности сети Петри при перенастройке параметров нейронной сети

На основе процессов, представленных на рисунке 6, можно составить матрицы инцидентности и согласно им сформировать сети Петри. Сгенерированные матрицы инцидентности сетей Петри имеют 8 строк, согласно количеству выходов нейронной сети, и определенное количество столбцов, соответствующее количеству шагов формирования сети Петри. В настоящем случае матрицы инцидентности имеют вид:

$$
\begin{array}{ccccrcccrcccccc} 
& t_{1} & t_{2} & t_{3} & & t_{1} & t_{2} & t_{3} & & t_{1} & t_{2} & t_{3} & t_{4} & t_{5} & t_{6} \\
t p_{0} & -1 & 0 & 0 & t p_{0} & -1 & 0 & 0 & t p_{0} & -1 & 0 & 0 & 0 & 0 & 0 \\
t p_{1} & +1 & -1 & 0 & t p_{1} & +1 & -1 & +1 & t p_{1} & +1 & -1 & 0 & 0 & 0 & 0 \\
t p_{2} & +1 & -1 & +1 & t p_{2} & +1 & -1 & 0 & t p_{2} & +1 & -1 & +1 & -1 & 0 & 0 \\
t t p_{3} & 0 & +1 & -1 & \left|A_{L 2}\right|=t p_{3} & 0 & +1 & -1 & \left|A_{L 3}\right|=t p_{3} & 0 & +1 & 0 & -1 & 0 & 0 \\
t p_{4} & 0 & +1 & -1 & t p_{4} & 0 & +1 & -1 & t p_{4} & 0 & +1 & -1 & 0 & 0 & 0 \\
t p_{5} & 0 & 0 & 0 & t p_{5} & 0 & 0 & 0 & t p_{5} & 0 & 0 & 0 & +1 & -1 & 0 \\
t p_{6} & 0 & 0 & 0 & t p_{6} & 0 & 0 & 0 & t p_{6} & 0 & 0 & 0 & 0 & +1 & -1 \\
t p_{7} & 0 & 0 & 0 & t p_{7} & 0 & 0 & 0 & t p_{7} & 0 & 0 & 0 & 0 & 0 & +1
\end{array}
$$

$\begin{array}{ccccccc} & t_{1} & t_{2} & t_{3} & t_{4} & t_{5} & t_{6} \\ t p_{0} & -1 & 0 & 0 & 0 & 0 & 0 \\ t p_{1} & +1 & -1 & 0 & 0 & 0 & 0 \\ t p_{2} & +1 & -1 & +1 & -1 & 0 & 0 \\ t A_{L 3} \mid=t p_{3} & 0 & +1 & 0 & -1 & 0 & 0 \\ t p_{4} & 0 & +1 & -1 & 0 & 0 & 0 \\ t p_{5} & 0 & 0 & 0 & +1 & -1 & 0 \\ t p_{6} & 0 & 0 & 0 & 0 & +1 & -1 \\ t p_{7} & 0 & 0 & 0 & 0 & 0 & +1\end{array}$

Согласно сформированным матрицам инцидентности на рисунке 5 представлены соответствующие сети Петри. При этом сети, представленные на рисунках 5a и 5b, были окончательно не сформированы из-за перенастройки нейронной сети при наличии сигнала «неверной операции». Окончательно автоматически синтезированная сеть Петри, которая должна отражать адекватный алгоритм настройки координирующей системы автоматического управления, визуализирована согласно инцидентной матрице и дополнительными условиями срабатывания переходов на рисунке 5с. Представленные на рисунке сети Петри «живые», без конфликтных ситуаций. Условие срабатывания определенного перехода в данных сетях Петри следующие:

$$
\forall p_{i} \in I\left(t_{i}\right): \mu\left(p_{i}\right)=1 \cup J_{i}>J_{\max }
$$


т. е, все входные позиции перехода должны содержать маркер, и при этом значение критерия качества работы системы , $\mathrm{i}=1 ; 2$, должно быть больше . Следует отметить, что критерий связан с функционированием 1-го координирующего уровня системы, а связан с верхним стабилизирующим уровнем КСАУ [8].

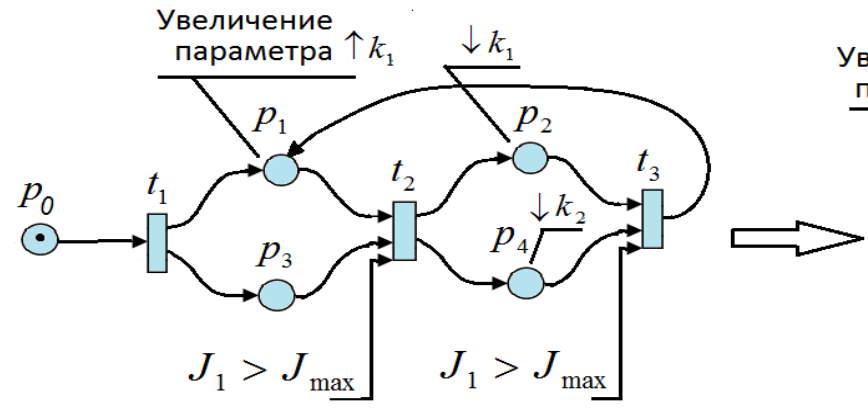

a)

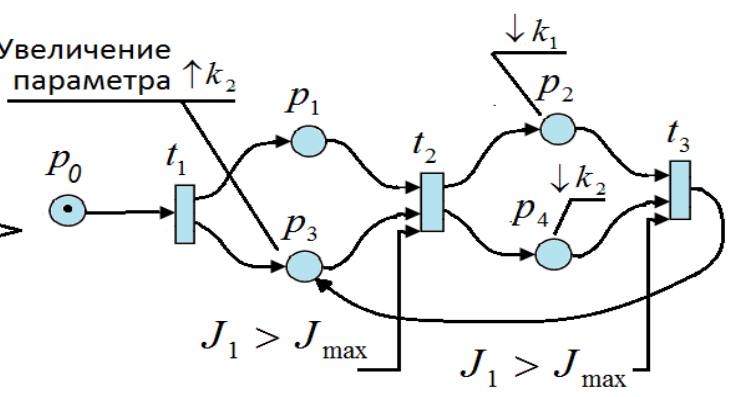

b)

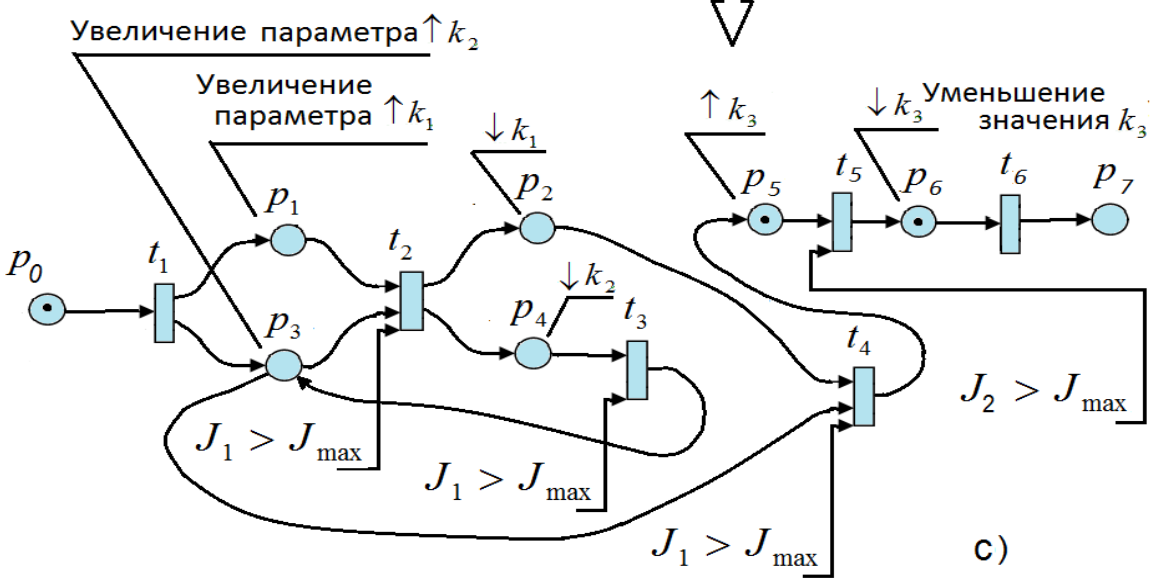

Рис. 7 - Визуализация сформированных сетей Пери при функционировании, и перенастройке параметров нейронной сети

Из рисунка 7 видно, что формируемые сети Петри представляют определенные алгоритмы, которые отражают последовательность необходимых операций при настройке системы, однако данные алгоритмы не представляют операции условного перехода "IF - THEN", тем самым указывая необходимость развития системы предусматривающей возможность композиции соответствующих сетей Петри.

Выводы

Представленный в настоящей работе алгоритм настройки определенной нейронной сети с исходными нейронами дает возможность выполнить определенный шаг в разработке интеллектуальной системы, формирующей алгоритмы управления объектами при автоматическом синтезе и композиции сетей Петри. Автоматически синтезируемая сеть Петри позволяет представить результат формирования алгоритма управления объектом и тем самым позволяет выработать специалисту, при необходимости, нужную корректировку данного алгоритма.

Следует отметить, что в данной разрабатываемой интеллектуальной системе, связанной с автоматическим синтезом сетей Петри, была представлена попытка использования принципа обучения с подкреплением, отражающего активно развивающуюся область искусственного интеллекта, нейросетевого моделирования и управления. Тем самым, развиваемая интеллектуальная система, связанная с синтезом сетей Петри и с формированием алгоритмов настройки особого класса систем управления, в достаточной мере вписывается в рамки современного развития интеллектуальных технологий, особенно актуальных в настоящее время.

Литература

[1] D. W. He, B. Strege, H. Tolle , A. Kusiak Decomposition in automatic generation of Petri nets for manufacturing system control and scheduling / DOI: 10.1080/002075400188942 // International Journal of Production Research 2000 - Volume 38, Issue 6, - pages 1437-1457

[2] Александров, А. В. Генерация конечных автоматов для управления моделью беспилотного самолета [Текст] / А. В. Александров, и др. // Научно-технический вестник информационных технологий, механики и оптики. 2011. - №. 2 (72). - C. 3-8.

[3] Филимонов, А. Б. О проблематике синтеза координирующих систем автоматического управления [Текст] / А. Б. Филимонов, Н. Б. Филимонов // Известия Южного федерального университета. Технические науки. -2012. - Т. 128. - №. 3.- С. 172-180. 
http://www.atbp.onaft.edu.ua/

[4] Гурский А. А. Координирующая система автоматического управления приводами робота-манипулятора / А.А. Гурский, А.Е. Гончаренко, С.М. Дубна // Автоматизация технологических и бизнес-процессов. - Одесса: 2016. - №4. - С. 11-21.

[5] Питерсон Дж. Теория сетей Петри и моделирование систем. - М. : Мир 1984. - 264 с.

[6] Харитонов Д.И. Метод генерации примеров моделей программ в терминах сетей Петри / Д.И. Харитонов, Е.А. Голенков, Г.В. Тарасов, Д.В. Леонтьев // Моделирование и анализ информационных систем. - 2015. - №4. - С. 563-577. DOI:10.18255/1818-1015-2015-4-563-577

[7] Ezpeleta Joaquín. Automatic Synthesis of Colored Petri Nets for the Control of FMSIn / Ezpeleta Joaquín, Colom José Manuel // IEEE Transactions on Robotics and Automation - June 1997. - Vol. 13, no. 3, - pages 327-337.

[8] Gurskiy, A. A. Formation of the synthesis algorithms of the coordinating control systems by means of the automatic generation of Petri nets [Text] / A. A. Gurskiy, A. E. Goncharenko, S. M. Dubna // Automation of technological and business processes. - Volume 8, Issue 3/2016 - P. 13-23.

[9] Нестерюк О.Г. Інформаційна технологія моделювання і аналізу дискретно-неперервних автоматизованих систем управління : автореф. дис. на здобуття наукового ступеня канд. тех. наук : спец.05.13.06 «Інформаційні технології» / О.Г. Нестерюк. - Одесса, 2016. - 23 с.

[10]Гурский А.А. Генерация сети Петри на базе средств дискретно-непрерывных сетей при формировании алгоритма автоматической настройки координирующей системы управления / А.А. Гурский, А.Е. Гончаренко, А.В. Денисенко // Электротехнические и компьютерные системы. - Одесса: 2017. - №26(102). - С. $78-87$.

[11]Саттон Р. С. Обучение с подкреплением / Р.С. Саттон, Э.Г. Барто. - М.: Бином, 2011. - 380 с.

\section{References}

[1] He, D. W., Strege, B., Tolle, H., Kusiak, A., (2000) Decomposition in automatic generation of Petri nets for manufacturing system control and scheduling. International Journal of Production Research, Volume 38, Issue 6, pages $1437-1457$.

[2] Aleksandrov, A. V. (2011) Generacija konechnyh avtomatov dlja upravlenija model'ju bespilotnogo samoleta [Generation of state machine creation for unmanned airplane controlling]. Scientific and Technical Journal of Information Technologies, Mechanics and Optics, vol. 2(72), pp. 3-8.

[3] Filimonov, A. B., Filimonov N. B. (2012). Concerning the problems of synthesis of coordinated systems of automatic control [O problematike sinteza koordiniruyuschih sistem avtomaticheskogo upravleniya] Izvestiya SfedU, Engineering sciences, no. 3, pp. 172-180.

[4] Gurskij A. A., Goncharenko, A. E., Dubna S. M. (2016) Koordinirujushhaja sistema avtomaticheskogo upravlenija privodami robota-manipuljatora [Coordinating automatic control systems of the robot manipulator drivers]. Avtomatizacija tehnologicheskih i biznes-processov - Automation of technological and business processes, no. 4, pp. 11-21.

[5] Peterson J. L. (1984) Teorija setej Petri i modelirovanie sistem [Petri net theory and the modeling of systems]. Moscow, Mir. 264 p.

[6] Kharitonov D.I., Golenkov E.A., Tarasov G.V., Leontyev D.V. (2015) Metod generacii primerov modelej programm v terminah setej Petri [A Method of Sample Models of Program Construction in Terms of Petri Nets]. Modelirovanie i analiz informacionnyh system - Modeling and Analysis of Information Systems, no. 4, pp. 563-577.

[7] Ezpeleta Joaquín, Colom José Manuel. (1997) Automatic Synthesis of Colored Petri Nets for the Control of FMSIn // IEEE Transactions on Robotics and Automation, vol. 13, no. 3, pp. 327-337.

[8] Gurskiy, A. A., Goncharenko A. E., Dubna S. M. (2016) Formation of the synthesis algorithms of the coordinating control systems by means of the automatic generation of Petri nets. Automation of technological and business processes, Volume 8, Issue 3, pp. 13-23.

[9] Nesteryuk, O.G. (2016). Informacijna tekhnologiya modelyuvannya i analizu diskretno-neperervnih avtomatizovanih sistem upravlinnya [Information technology modelling and analysis of discrete-continuous automated control systems]. Extended abstract of candidate's thesis. Odessa [in Ukraine].

[10]Gurskij, A. A., Goncharenko, A. E., Denisenko, A.V. (2017) Generaciya seti Petri na baze sredstv diskretnonepreryvnyh setej pri formirovanii algoritma avtomaticheskoj nastrojki koordiniruyushchej sistemy upravleniya [Generation of the Petri net by means of the resource of the discrete-continuous nets in the algorithm formation for the self-tuning of the coordinating control system] Electrotechnic and Computer Systems, no. 26(102), pp. $78-87$.

[11] Satton, R.S., Barto, JE. G. (2011). Obuchenie s podkrepleniem [Reinforcement Learning] Moscow, Binom. 380 p. 